\title{
Effectiveness of Previous Mumps Vaccination during the 2014-2015 Outbreak in Lebanon
}

Nadine Haddad ${ }^{1 *}$, Hala Abou Naja ${ }^{1}$, Sabine Kassouf ${ }^{1}$, Adela Paez Jimenez ${ }^{2}$, Ghada Abou Mrad ${ }^{1}$, Walid Ammar $^{1}$ and Nada Ghosn $^{1}$

${ }^{1}$ Ministry of Public Health, Republic of Lebanon

2 MediPIET at European Centre for Disease Prevention and Control (ECDC), Sweden

*Corresponding author: Nadine Haddad, Ministry of Public Health, Republic of Lebanon, E-mail: esu.haddadn@gmail.com

Received date: December 01, 2017; Accepted date: December 13, 2017; Published date: December 20, 2017

Copyright: $\odot 2017$ Haddad N, et al. This is an open-access article distributed under the terms of the Creative Commons Attribution License, which permits unrestricted use, distribution, and reproduction in any medium, provided the original author and source are credited.

\begin{abstract}
Introduction: In Lebanon, MMR was introduced at 12 months and 4-5 years in 1996. In 2014, the 2nd MMR dose was shifted to 18 months. Despite a national MMR coverage estimated at $79 \%$, a six-fold increase in mumps national incidence was observed in December 2014. The objective of this study is to determine mumps vaccine effectiveness (VE) among Lebanese population to guide immunization policies.

Methods: Clinical and confirmed mumps cases reported to the Epidemiological Surveillance Program between 2014W46 and 2015W11 were eligible if they were Lebanese and aged 1.5 to 19 years-old. Controls were randomly selected controls using phonebook of the same area and matched 1:1 on age and locality. Information was collected by structured phone interviews. Mumps vaccination status was based on documented valid dates for MMR doses. Data were entered using Epidata 3 and analyzed using Stata13. VE ((1-OR) $\times 100)$ of one and two doses and ORs $(95 \% \mathrm{Cl})$ for acquiring mumps were estimated using conditional logistic regression.
\end{abstract}

Results: 91 cases and 91 controls were included. Only $36 \%$ of cases had vaccination cards, compared to $71 \%$ of controls $(p<0.001) .94 \%$ of cases were not vaccinated compared to $51 \%$ of controls $(p<0.001)$. Vaccine effectiveness was estimated $60 \%(\mathrm{Cl}=-27 \%: 88 \%)$ for one dose and $88 \%(\mathrm{Cl}=60: 96 \%)$ for 2 doses.

Conclusion: Two-doses of MMR vaccine were estimated as $88 \%$ effective against mumps, similar to results found in the literature. This outbreak can be explained by suboptimal MMR2 coverage. Efforts should focus on achieving high MMR coverage and raising population's awareness about preserving documentation of vaccination.

Keywords Mumps; Vaccine effectiveness; Case-control; Lebanon

\section{Introduction}

Mumps is a viral contagious infection transmitted from one person to another by direct contact, droplets or contaminated fomites. The incubation period is about 15-24 days median 19 days) [1]. A person infected with mumps is mostly contagious 1-2 days before the onset of clinical symptoms until 7-8 days afterwards. The disease is characterized by a painful swelling of the parotid gland, which occurs in $60-70 \%$ of infections. About a third of mumps infections arise without recognized symptoms. Clinically manifest infections might start with a short prodromal phase of low-grade fever, anorexia, malaise and headache. Aseptic meningitis and encephalitis (up to $10 \%$ of infections) and encephalitis (1\%) are common complications of mumps together with orchitis in adult men; other complications include deafness and pancreatitis [2]. Mumps vaccines consist of live attenuated virus and are available as monovalent vaccine or most often in combination with measles and rubella vaccines (MMR). In the late 1960 s', the original randomized clinical trials yielded efficacy estimates of more than $95 \%$ for the monovalent vaccine containing the Jeryl Lynn strain [3]. Vaccine effectiveness is assessed, after a vaccine has been introduced into general use, commonly in the occasional outbreak situations. Thus, mumps vaccine effectiveness of prior vaccination with one dose of vaccine ranged $64-80 \%$ and $88-95 \%$ for two doses in outbreaks studies [4-8]. Of note over the last years, large outbreaks have indeed affected highly vaccinated populations in England [7], Germany [9], Belgium [10], Netherlands [11], or the US $[5,8,12-14]$. In Lebanon, vaccination against mumps was initiated since 1996 by administering a first dose of measles-mumps-rubella (MMR) vaccine at 12 months of age, and a second dose at 4-5 years. In 2014, the timing of the second dose was shifted to 18 months [15]. In parallel, the monovalent vaccine is administered in the public health sector at 9 months of age.

Therefore, the vaccination coverage for mumps is assessed by the second dose of the measles containing vaccine (MCV2, which can be MMR1 or MMR2 depending on the provider). Before 2007, mumps national vaccination coverage defined at that time as first dose of MMR (MMR1), was below 50\% according to the official national administrative estimates. This coverage increased to $71 \%$ in 2008 , ranged between 86 to $89 \%$ between 2009 and 2012, and started decreasing in 2013 to reach $69 \%$ in 2014 [16]. On the other hand, MMR1 coverage was estimated by WHO-UNICEF at $85 \%$ in 2008 , and remained 75\% between 2009 and 2014 [17]. To date, no studies on the effectiveness of a Mumps-containing vaccine were conducted in Lebanon. In Lebanon, identification of a suspected mumps case requires immediate notification to the Epidemiological Surveillance Program at the Ministry of Public Health (ESUMOH). Information on reported mumps cases is entered in the national ESUMOH database. Classification relies on clinical and laboratory findings. A suspected case of mumps is defined by the acute onset of unilateral or bilateral 
tender and self-limited swelling of the parotid or other salivary gland lasting 2 or more days without other apparent cause. A confirmed case is defined by positive results for at least one of the following tests: virus isolation, positive serological testing for mumps-specific IgM antibodies or at least fourfold rise in serum mumps IgG titter in the absence of mumps immunization in the preceding six weeks. Since laboratory confirmation for mumps is not routinely performed in Lebanon, most of the reported cases are suspected cases. Over the last decade, national mumps incidence decreased from 5 per 100,000 in 2007 to less than 1 in 2010 until 2013. In 2014, Lebanon was hit by a national outbreak with an incidence of 12.74 , which increased to 23.3 in 2015. Highest incidence rates, greater than 57 per 100,000 were recorded in the provinces of Bekaa and North Lebanon.

Of interest, children 5-9 years old were the most affected (52 per $100,000)$, followed by the age group $10-19$ years $(46$ per 100,000) and $0-4$ years $(27$ per 100,000$)$. Around $83 \%$ of the cases reported in this outbreak are between 1 and 19 years old, who are eligible to receive at least one MMR dose. According to the reporting forms around $36 \%$ of these cases are vaccinated (and 30\% have an unknown vaccination status). However, this information is recorded by the healthcare provider without verification of the vaccination card. Therefore, such proportion of reported vaccinated cases (36\%) is not validated. In this context, understanding the risk factors for mumps occurrence and estimating the mumps vaccine effectiveness were deemed necessary in order to guide immunization policies and appropriate interventions.

\section{Materials and Methods}

A case-control study was conducted among Lebanese reported Mumps cases, and community controls matched $1: 1$ by age and locality of residence.

\section{Selection of cases and controls}

A case was defined as a suspected or a lab-confirmed mumps case, registered in ESUMOH central database, with a date of onset between 2014W46 and 2015W11. Only Lebanese cases, between 1.5 and 19 years old at time of onset, and having a phone number were included. Suitable controls were identified through an iterative selection process starting with systematic random sampling from the latest version of the national phonebook (version 2005). Matching was conducted for locality of residence and age ( \pm 1 year for cases less than 5 years old, and up to \pm 4 year for cases greater than 10 years old). Controls were eligible if they were Lebanese, never been diagnosed with mumps, and not being in contact with a mumps case during the 25 days preceding the phone interview.

\section{Data collection}

A structured questionnaire was orally administered through phone call interviews conducted by local ESUMOH staff. Collected variables included socio-demographic information (as highest educational level of study participant and mother, number of persons living in the household, number of household rooms...) and also vaccination status and illness history. As for mumps vaccination status, respondents (who were mainly parents given the young age range of study subjects) were asked to report whether the subject was vaccinated against mumps. Subsequently, they were asked to provide their vaccination card and the reported vaccination history was verified against it. To further assess the vaccination status of subjects reporting not having any vaccination card, they were asked about their place of vaccination (if any, whether in public or private sector) and the reason for not being vaccinated.

\section{Sample size calculation}

The sample size to estimate Mumps vaccine effectiveness was calculated using the free software OpenEpi [11]. Assuming 75\% exposure prevalence among controls (based on WHO estimated MMR1 coverage), $80 \%$ power and $95 \%$ confidence interval, 94 cases and 94 controls were needed to detection a minimal odds ratio of 0.4 .

\section{Data analysis}

Epidata v.3 was used for data entry and Stata v13 for data cleaning and analysis. The variable "crowding index" was generated as the number of persons living in the same household divided by the number of household rooms. Comparison between cases and controls were conducted using independent samples t-test for means and McNemar's test for frequencies. The odds ratios and 95\% confidence intervals were calculated using the conditional logistic regression. The variable "ever being vaccinated" was generated as follows: Study subjects for whom a vaccination card was provided were classified as having a "documented vaccination status".

Among them, only those with the vaccination date specified in the card were considered "vaccinated against mumps". On the other hand, study subjects were considered "not vaccinated against mumps" if they reported not being vaccinated at all, or not having a vaccination card, or having a vaccination card lacking vaccination dates. This categorization aimed to rely only on documented vaccination status in attempt to prevent any recall or reporting bias from the participants. Subsequently, mumps vaccine effectiveness was calculated for one and two doses using the following formula: VE $(\%)=(1-\mathrm{OR}) \times 100$, among those study subjects fulfilling the "vaccinated against Mumps" criteria afore mentioned.

\section{Results}

One hundred thirty-three eligible cases with dates of onset between 2014W46 and 2015W11 were identified, of whom 41 were not reachable and 1 refused to participate in the study. In total, 91 cases and 91 controls between 4 and 20 years old were enrolled. Around $4 \%$ of the cases reported orchitis. There were no significant differences between cases and controls with respect to age (11 years \pm 4.11 , $\mathrm{p}=0.42)$ and sex (53\% males, $\mathrm{p}=0.76)$. About $90 \%$ were students $(\mathrm{p}=0.07)$, and almost half of them have the primary level as their highest educational level $(\mathrm{p}=0.64)$. Cases $(55 \%)$ were significantly more likely than controls $(12 \%)$ to have mothers with a primary educational level or below $(\mathrm{p}<0.001)$ (Table 1$)$. In addition, household crowding index was significantly higher among cases $(1.7 \pm 0.7)$ than among controls $(1.1 \pm 0.6)(\mathrm{p}<0.001)$ (Table 1$)$. 
Citation: Haddad N, NajaHA, Kassouf S, Jimenez AP, Mrad GA, et al. (2018) Effectiveness of Previous Mumps Vaccination during the 2014-2015 Outbreak in Lebanon. J Vaccines Vaccin 9: 378. doi:10.4172/2157-7560.1000378

Page 3 of 6

\begin{tabular}{|c|c|c|c|}
\hline Age (in years) & $11.4 \pm 4.06$ & $10.9 \pm 4.17$ & 0.419 \\
\hline \multicolumn{4}{|l|}{ Sex } \\
\hline Male & $54(59 \%)$ & $52(57 \%)$ & 0.764 \\
\hline Female & $37(41 \%)$ & $39(43 \%)$ & \\
\hline \multicolumn{4}{|l|}{ Occupational status } \\
\hline Non student & $6(7 \%)$ & $3(3 \%)$ & 0.305 \\
\hline Student/employee & $85(93 \%)$ & $88(97 \%)$ & \\
\hline \multicolumn{4}{|c|}{ Highest educational level } \\
\hline Kindergarten & $11(12 \%)$ & $8(9 \%)$ & 0.345 \\
\hline Primary & $46(51 \%)$ & $40(45 \%)$ & \\
\hline Intermediate & $25(27 \%)$ & $27(31 \%)$ & \\
\hline Secondary & $7(8 \%)$ & $12(14 \%)$ & \\
\hline University & $2(2 \%)$ & $1(1 \%)$ & \\
\hline \multicolumn{4}{|c|}{ Mother's highest educational level ( 2 categories) } \\
\hline Primary or less & $47(55 \%)$ & $10(12 \%)$ & $<0.001$ \\
\hline Intermediate or more & $39(45 \%)$ & $73(88 \%)$ & \\
\hline Crowding index & $1.8 \pm 0.07$ & $1.2 \pm 0.05$ & $<0.001$ \\
\hline
\end{tabular}

Table 1: Description of Cases and Controls according to demographic characteristics.

Almost half of the cases (53\%) verbally reported being vaccinated against mumps, as compared to $97 \%$ among controls $(\mathrm{p}<0.001$ ) (Table 2 ). However, the documentation of the vaccination status was available for $36 \%$ of the cases $(n=33)$ and $71 \%$ of the controls $(n=63)(p<0.001)$. Of interest, the availability of the vaccination card was not dependent on the age. A significant association $(\mathrm{p}<0.001)$ was found between mother's educational level and both verbally-reported vaccinated child, and documented-with- card vaccinated child. For instance, around $55 \%$ of subjects verbally reported as not vaccinated or without a vaccination card had mothers with only primary education. The classification of mumps vaccinated status was limited to subjects with vaccination cards. "Zero mumps dose" was found for 16 cases and 12 controls, "One dose" for 8 cases and 12 controls, "Two doses" for 4 cases and 30 controls, "Three doses" for 9 cases and 7 controls $(\mathrm{p}<0.001)$ (Table 2). The mean age at second mumps vaccination was significantly higher for cases $(6.2 \pm 3.7)$ than controls $(4.2 \pm 1.4)$ $(\mathrm{p}=0.03)$.

\begin{tabular}{|l|l|l|l|}
\hline Mumps vaccination & $\begin{array}{l}\text { Cases } \\
(\mathbf{N}=\mathbf{9 1})\end{array}$ & $\begin{array}{l}\text { Controls } \\
(\mathbf{N}=\mathbf{9 1})\end{array}$ & P-value \\
\hline Reported as vaccinated & & & \\
\hline Yes & $48(53 \%)$ & $88(97 \%)$ & $<0.001$ \\
\hline No/ Unknown & $43(38 \%)$ & $3(3 \%)$ & \\
\hline Vaccination card available & & & $<0.001$ \\
\hline Yes (documented status) & $33(36 \%)$ & $63(71 \%)$ & $26(29 \%)$ \\
\hline No/Unknown (not documented) & $58(64 \%)$ & & $12(13 \%)$ \\
\hline Number of doses & & $12(13 \%)$ & - \\
\hline 0 & $16(43 \%)$ & $8(22 \%)$ & 0.242 \\
\hline 1 & & & \\
\hline
\end{tabular}


Page 4 of 6

\begin{tabular}{|l|l|l|l|}
\hline 2 & $4(11 \%)$ & $32(33 \%)$ & $<0.001$ \\
\hline 2 or more & $9(24 \%)$ & $39(41 \%)$ & 0.954 \\
\hline Mean age at first dose & $1.8 \pm 1.4(n=11)$ & $1.3 \pm 0.9(n=41)$ & 0.1782 \\
\hline Mean age at second dose & $6.2 \pm 3.7(n=5)$ & $4.2 \pm 1.38(n=31)$ & 0.0307 \\
\hline
\end{tabular}

Table 2: Description of Cases and Controls according to their Mumps vaccination.

Considering only subjects with documented vaccination dates, mumps vaccine effectiveness was calculated at $60 \%$ (95\%CI: $(-27 \%$; $88 \%)$ ) for one mumps dose and $88 \%$ (95\%CI: (63\%; 95\%)) for two doses (Table 3$)$. The crude odds ratio for cases ever being vaccinated against mumps as compared to controls was 0.11 (95\%CI: (0.03-0.38)) $(\mathrm{p}<0.001)$, resulting in a crude overall vaccine effectiveness of $82 \%$ (95\% CI: $57 \%-93 \%$ ). Calculated by the number of doses, the crude vaccine effectiveness was calculated at $60 \%$ (95\%CI: $(-27 \%$; $88 \%))$ for one dose and $88 \%$ (95\%CI: (62\%; 97\%)).

\begin{tabular}{|c|c|c|c|c|c|}
\hline Mumps vaccination & Cases & Control & $\begin{array}{l}\text { Matched } \\
\text { OR }\end{array}$ & VE (\%) & $95 \% \mathrm{Cl}$ \\
\hline $\begin{array}{l}\text { Overall Mumps } \\
\text { vaccine } \\
\text { effectiveness }\end{array}$ & & & & & \\
\hline Ever vaccinated & $13(14 \%)$ & $40(44 \%)$ & 1 & & \\
\hline Not vaccinated & 78 (86\%) & $51(56 \%)$ & 0.18 & $82 \%$ & $\begin{array}{l}(57 \%-9 \\
3 \%)\end{array}$ \\
\hline $\begin{array}{l}\text { Vaccine } \\
\text { effectiveness by } \\
\text { number of doses }\end{array}$ & & & & & \\
\hline Not vaccinated* & 76 & 46 & - & - & \\
\hline 1 dose & 8 & 12 & 0.4 & $60 \%$ & $\begin{array}{l}(-27 \% \\
88 \%)\end{array}$ \\
\hline 2 doses & 4 & 30 & 0.12 & $88 \%$ & $\begin{array}{l}(62 \% ; \\
97 \%)\end{array}$ \\
\hline
\end{tabular}

"Not vaccinated is defined here as: reported as not vaccinated, or without vaccination card, or having a vaccination card with no vaccination dates

Table 3: Mumps vaccine effectiveness calculation.

\section{Discussion}

The eleven-fold increase in the national mumps incidence in 2014 was preceded by low MCV2 coverage estimates over the previous years. The consequent accumulation of susceptible population may explain this 2014-2015 mumps outbreak in Lebanon, as evidenced by the significant difference in the proportion of cases (65\%) and controls (26\%) non properly vaccinated, i.e. with less than two doses. The study was not designed to look at vaccine effectiveness by age groups and in the absence of data on administrative vaccination coverage by age or birth cohort, it is difficult to argue further. It should also be considered MCV2 coverage does not equate MMR2 in Lebanon, since monovalent mumps vaccine was also administered. Since only $76 \%$ of the controls in this study were vaccinated with two doses, herd immunity of these communities is less than optimum to block the spread of the outbreak in high-risks settings for exposure such as schools or overcrowded areas. Of note, Anderson and May [18] and models developed later
[19] suggested an $88-92 \%$ herd immunity threshold to prevent mumps community transmission and outbreaks.

Indeed, living with a large number of housemates increased the risk of mumps infection; high population density provides increased opportunities for close contact and higher dose exposure to mumps virus. As mentioned before, most of recent large mumps outbreaks in highly vaccinated population have occurred in communal living situations, such as dormitories or boarding schools $[12,20]$.

Mother's low level of education was also associated with an increased risk of mumps. It may be related to lack of awareness of vaccine as our most effective technology to prevent infectious diseases or of where and how to get them for free. Similar findings were also documented in studies on socio-demographic characteristics of MMR vaccination uptake in France [21] and Belgium [22].

Another factor contributing to the moderate effectiveness of the two-dose mumps vaccine ( $88 \%$ in this study) could be related to the combination of vaccine strains used in Lebanon, since Jeryl-Lynn strain seems to offer greater protection than the Urabe strain $(80.7 \%$ versus 54.4\%) [23]. Unfortunately, the vaccine strain administered was almost never documented in the vaccination card and vaccine strains used in the public and private sector may differ. All in all, the Jeryl Lynn strain most widely used as the mumps component in MMR vaccine is derived from genotype $A$ and less effective against serogroup G wild strains [24] currently circulating in the Middle East [25].

Last, potential bias due to nonrandom distribution of outbreak sizes and resulting in the underestimation of vaccine effectiveness has been discussed as a common drawback in outbreak studies. Precisely, outbreak investigations will tend to underestimate vaccination efficacy to an extent which is related directly to the size of the epidemic, the vaccination coverage in the community, and the extent of clustering of vaccination failures in the population and inversely related to the size of and contact intensity within the investigated community [26]. Our study may suffer from a limited selection bias regarding both disease and exposure. Some misclassification of mumps cases may have happen, as most of the cases participating in the study are clinical cases with no laboratory confirmation. Other viral infections (Epstein-Barr virus, parainfluenza virus 1 and 3, influenza a virus, coxsackievirus, adenovirus, parvovirus B19) can also cause parotitis [27] but not on an epidemic scale; such etiologies are rather considered when the incidence rate of mumps is low. Thus, if here was any misclassification of cases it should have been very small. Regarding misclassification of the exposure, only study participants with vaccination card available where considered as vaccinated. Since cases seem slightly more prone not having their vaccination card, this may have led to an overestimation of the vaccine effectiveness.

A curious finding is the one-year delayed second dose of mumps vaccination among cases; however, in view of the low number of cases involved it cannot be interpreted. In Lebanon, the second dose of 
Page 5 of 6

MMR was shifted from 5-6 years old to 18 months in 2014. An increased in the proportion of unvaccinated individuals and those vaccinated with only one dose a year after the change in the booster schedule has been described elsewhere [28]. All in all with most countries nowadays using a routine two-dose schedule but very large variation in the at the age of second dose [2], the ideal schedule for mumps vaccination in different settings is an unresolved issue which deserves major attention.

\section{Conclusion and recommendations}

Despite the several limitations of our study, it suggests the suboptimal uptake of the MMR vaccine can explain the 2014-2015 mumps outbreak in Lebanon. In addition, the demonstrated unavailability of vaccination cards is a critical issue to verify vaccination status.

To prevent future mumps outbreaks population awareness about the importance of mumps vaccination should be raised. Also, routine MMR (1 and 2) vaccination is to be strengthened targeting unvaccinated clusters of population to avoid growing pockets of susceptible population. This outbreak is a wake-up call to the growing numbers of children and young people not adequately protected in Lebanon. In order to get ahead of mumps and measles instead of chasing it, a national catch-up campaign could be considered. In addition, there is a substantial need to raise awareness about the importance of keeping the vaccination card even if one had completed all essential vaccinations. Establishing electronic immunization records is a possible opportunity for a better assessment of the vaccination status and gaps in the general population. Finally, strengthening laboratory capacities in the country are critical in order to confirm future outbreaks.

\section{Acknowledgments}

The authors would like to thank all the participants for their willingness and time; Fatmeh Farhat, Hiba Souki, Itab Kaysar, Laura Keyrouz , Rania Sarkis and Sabine Kassouf for their assistance with data collection; Dr Dragan Lausevic for feedback on study design and interpretation of results. Contributors: All authors contributed to the conception and design of the study, including the plan for statistical analysis and also to the interpretation of findings; $\mathrm{NH}$ performed the statistical analysis and drafted the manuscript; all authors were involved in the critical review and had final approval of the manuscript. Conflict of interest statement: The authors declare no conflict of interests. Funding: This work was entirely supported by the Epidemiological Surveillance Program of the Ministry of Public Health, Republic of Lebanon (ESUMOH).

\section{References}

1. Richardson M, Elliman D, Maguire H, Simpson J, Nicoll A (2001) Evidence base of incubation periods, periods of infectiousness and exclusion policies for the control of communicable diseases in schools and preschools. Pediatr Infect Dis J 20: 380-391.

2. Hviid A, Rubin S, Muhlemann K (2008) Mumps. Lancet 371: 932-944.

3. Hilleman MR, Weibel RE, Buynak EB, Stokes J, Whitman JE (1967) Live attenuated mumpsvirus vaccine. IV. Protective efficacy as measured in a field evaluation. N Engl J Med 276: 252-258.

4. Harling R, White JM, Ramsay ME, Macsween KF, van den Bosch C (2005) The effectiveness of the mumps component of the MMR vaccine: a case control study. Vaccine 23: 4070-474.
5. Schaffzin JK, Pollock L, Schulte C, Henry K, Dayan G, et al. (2007) Effectiveness of previous mumps vaccination during a summer camp outbreak. Pediatrics 120: e862-868.

6. Sartorius B, Penttinen P, Nilsson J, Johansen K, Jonsson K, et al. (2005) An outbreak of mumps in Sweden, February-April 2004. Euro surveillance : bulletin Europeen sur les maladies transmissibles = European communicable disease bulletin 10: 191-193.

7. Cohen C, White JM, Savage EJ, Glynn JR, Choi Y, et al. (2007) Vaccine effectiveness estimates, 2004-2005 mumps outbreak, England. Emerg Infect Dis 13: 12-17.

8. Livingston KA, Rosen JB, Zucker JR, Zimmerman CM (2014) Mumps vaccine effectiveness and risk factors for disease in households during an outbreak in New York City. Vaccine 32: 369-374.

9. Takla A, Bohmer MM, Klinc C, Kurz N, Schaffer A, et al. (2014) Outbreak-related mumps vaccine effectiveness among a cohort of children and of young adults in Germany 2011. Hum Vaccin Immunother 10: 140-145.

10. Braeye T, Linina I, De Roy R, Hutse V, Wauters M, et al. (2014) Mumps increase in Flanders, Belgium, 2012-2013: results from temporary mandatory notification and a cohort study among university students. Vaccine 32: 4393-4398.

11. Greenland K, Whelan J, Fanoy E, Borgert M, Hulshof K, et al. (2012) Mumps outbreak among vaccinated university students associated with a large party, the Netherlands, 2010. Vaccine 30: 4676-4680.

12. Cortese MM, Jordan HT, Curns AT, Quinlan PA, Ens KA, et al. (2008) Mumps vaccine performance among university students during a mumps outbreak. Clin Infect Dis 46: 1172-1180.

13. Dayan GH, Quinlisk MP, Parker AA, Barskey AE, Harris ML, et al. (2008) Recent resurgence of mumps in the United States. N Engl J Med 358: 1580-1589.

14. Marin M, Quinlisk P, Shimabukuro T, Sawhney C, Brown C, et al. (2008) Mumps vaccination coverage and vaccine effectiveness in a large outbreak among college students--Iowa, 2006. Vaccine 26: 3601-3607.

15. Lebanon (2017) MoPHo. Expanded Program of Inmunization: National Immunization Calendar.

16. Lebanon (2017) MoPHo. "Surveillance data".

17. Lebanon (2017) MoPHo. "Notifiable communicable diseases.

18. Anderson RM, May RM (1985) Vaccination and herd immunity to infectious diseases. Nature 318: 323-329.

19. Edmunds WJ, Gay NJ, Kretzschmar M, Pebody RG, Wachmann H (2000) Network EPES-e. The prevaccination epidemiology of measles, mumps and rubella in Europe: implications for modelling studies. Epidemiol Infect 125: 635-650.

20. Brockhoff HJ, Mollema L, Sonder GJ, Postema CA, van Binnendijk RS, et al. (2010) Mumps outbreak in a highly vaccinated student population, The Netherlands, 2004. Vaccine 28: 2932-2936.

21. Rotily M, Guagliardo V, Fontaine D, Garros B, Mayer C, et al. (2001) [Evaluation of measles, mumps and rubella vaccine coverage in 3 year old children in twelve French counties. Time-trends and related factors]. Rev Epidemiol Sante Publique 49: 331-341.

22. Vandermeulen $C$, Roelants $M$, Theeten $H$, Van Damme $P$, Hoppenbrouwers K (2008) Vaccination coverage and sociodemographic determinants of measles-mumps-rubella vaccination in three different age groups. Eur J Pediatr 167: 1161-1168.

23. Ong G, Goh KT, Ma S, Chew SK (2005) Comparative efficacy of Rubini, Jeryl-Lynn and Urabe mumps vaccine in an Asian population. J Infect 51: 294-298.

24. Quinlisk MP (2010) Mumps control today. J Infect Dis 202: 655-656.

25. Jin L, Orvell C, Myers R, Rota PA, Nakayama T, et al. (2015) Genomic diversity of mumps virus and global distribution of the 12 genotypes. Rev Med Virol 25: 85-101.

26. Fine PE, Zell ER (1994) Outbreaks in highly vaccinated populations: implications for studies of vaccine performance. Am J Epidemiol 139: 77-90. 
Citation: Haddad N, NajaHA, Kassouf S, Jimenez AP, Mrad GA, et al. (2018) Effectiveness of Previous Mumps Vaccination during the 2014-2015 Outbreak in Lebanon. J Vaccines Vaccin 9: 378. doi:10.4172/2157-7560.1000378

Page 6 of 6

27. Barrabeig I, Costa J, Rovira A, Marcos MA, Isanta R, et al. (2015) Viral etiology of mumps-like illnesses in suspected mumps cases reported in Catalonia, Spain. Hum Vaccin Immunother 11:282-287.
28. Santacruz-Sanmartin E, Hincapie-Palacio D, Ospina MC, Perez-Toro O, Bernal-Restrepo LM, et al. (2015) Seroprevalence of mumps in an epidemic period in Medellin, Colombia.Vaccine 33: 5606-5612. 